Horizons philosophiques

Horizons

\title{
Pour une pensée conservatrice : contre l'éthique démocratique dans la compréhension de l'identité nationale québécoise
}

\section{Mathieu Bock-Côté}

Volume 12, numéro 2, printemps 2002

Valeurs et modernité

URI : https://id.erudit.org/iderudit/801208ar

DOI : https://doi.org/10.7202/801208ar

Aller au sommaire du numéro

Éditeur(s)

Collège Édouard-Montpetit

ISSN

1181-9227 (imprimé)

1920-2954 (numérique)

Découvrir la revue

Citer cet article

Bock-Côté, M. (2002). Pour une pensée conservatrice : contre l'éthique démocratique dans la compréhension de l'identité nationale québécoise. Horizons philosophiques, 12(2), 57-73. https://doi.org/10.7202/801208ar d'utilisation que vous pouvez consulter en ligne. 


\title{
POUR UNE PENSÉE CONSERVA- TRICE : CONTRE L'ÉTHIQUE DÉMOCRATIQUE DANS LA COMPRÉHENSION DE L'IDENTITÉ NATIONALE QUÉBÉCOISE
}

\author{
Pousser une idée jusqu'à ses conséquences logiques \\ extrêmes, c'est souvent, sous prétexte de l'accomplir, \\ la nier et même la faire périr. \\ Julien Freund \\ Un peuple qui défigure le réel parce qu'il n'a pas envie \\ de se trouver en face de la vérité nue devient vite la \\ victime effarée de ses propres élucubrations. \\ Chantal Millon-Delsol
}

La radicalisation de l'éthique démocratique est le fait politique majeur de notre temps. Conformément à l'intuition tocquevillienne, la dynamique égalitaire ne s'est pas arrêtée à refaçonner les institutions politiques selon le principe de la souveraineté populaire. La démocratie n'est plus qu'un régime politique. C'est maintenant un état de société intégral, où la dynamique égalitaire recrée systématiquement le réel à travers le langage de la reconnaissance des droits de chacun à une forme avancée d'authenticité. II était illusoire de croire que le principe démocratique, générateur de la dynamique égalitaire, serait endigué longtemps par les formes héritées du monde pré-démocratique. Anthony Giddens, dans une question à vocation programmatique, demandait dans The Third Way : "How can we democratize democracy" ?' Question centrale de l'éthique démocratique contemporaine, qui perçoit de façon aiguë le caractère inachevé de la démocratie. L'éthique démocratique nous conduit à lire dans la "démocratisation" un processus sans fin qui ne doit buter sur aucune forme d'autorité puisant à la même intuition qu'elle.

La plupart des enjeux au centre de l'espace public se redéfinissent selon les paramètres de cette exigence. La réforme du mode de scrutin dans une perspective proportionnelle, la parité homme-femme au sein de l'Assemblée nationale, la représentativité des différentes minorités culturelles au sein de l'administration publique, la création

1. Anthony Giddens, The Third Way, Polity Press, 1999, p.72. 
d'un revenu de citoyenneté, toutes ces exigences sont légitimées par un prétexte démocratique. Chaque fois, il s'agit d'accélérer le processus de démocratisation. Un processus qui ne se reconnaît d'autres bornes que la possibilité de son accomplissement dans une réalisation intégrale du principe égalitaire.

Le débat sur la nation québécoise s'est aussi inscrit dans ce paradigme. Lorsque Gérard Bouchard, figure emblématique de ce réformisme identitaire radical, progressiste et démocratique, dresse une mise en accusation contre les identités collectives "d'antan", supposées "monolithiques" et "autoritaires»2, il le fait au nom de la possibilité "de "créer" (c'est nous qui soulignons) démocratiquement de l'identité dans la diversité et la fragmentation ${ }^{3}$ ". II le fait au nom d'une création intégrale de l'identité selon la maxime un jour défini par Raoul Vaneigem : «le passé que je n'ai pas choisi m'indiffère». II questionne sans ambiguitté les identités collectives : "comment introduire plus de démocratie dans le processus d'institution de la culture publique ${ }^{4}$ ?

La démocratie prend ici le visage d'un respect intégral de l'éthique de la pluriethnicité5, censée permettre aux "voix dissonantes" au sein des communautés nationales de se faire entendre dans leur authenticité6. II n'y aurait de démocratie qu'à travers le respect préalable de la diversité culturelle $e^{7}$ propre à chaque communauté politique, cette dernière étant nécessairement une communauté dialogique polyphonique ${ }^{8}$. "La nation co-intégrée n'admet pas de hiérarchie entre les ethnies et les cultures ${ }^{\natural}$. James Tully affirme ainsi :

2. Gérard Bouchard, "Nation et co-intégration : contre la pensée dichotomique» in Repères en mutation, Identités et citoyenneté dans le Québec contemporain, Jocelyn Maclure et Alain G. Gagnon (dir.), Québec-Amérique, 2001, p. 21.

3. op. cit. p. 28. Gérard Bouchard parle aussi d'une uforme d'identité au sein d'une hétérogénéité composite et friable" (p. 29).

4. Gérard Bouchard, "Qu'est-ce qu'une nation?» In Nationalité, citoyenneté et solidarité, Montréal, Liber, 1999, p. 477.

5. Op. cit. p. 28.

6. C'est là la démarche engagée par Jocelyn Maclure, notamment dans Récits identitaires, Le Québec à l'heure du pluralisme, Montréal, Québec-Amérique, 2000.

7. On pourrait aisément poursuivre en disant : sociale, ethnique, économique, professionnelle...

8. Nous reprenons ici la langue de bois en vigueur dans les cénacles de la bonne pensée socialement acceptée au sein de l'Université québécoise et de l'espace médiatique.

9. Gérard Bouchard, "Nation et co-intégration : contre la pensée dichotomique», op. cit. p. 35. 
«une constitution peut chercher à imposer une pratique culturelle, une façon de suivre les règles, ou elle peut reconnaître une diversité de manières culturelles d'être un citoyen; mais elle ne peut éliminer, vaincre ou transcender cette dimension culturelle de la politique ${ }^{10}$ ". La démocratie présuppose donc un contexte culturel souvent éclaté, très éloigné de la représentation homogène du fait national. Faire taire une seule de ces cultures soit par une représentation tronquée de la nation, soit par des pratiques «discriminatoires" à prétention universaliste ${ }^{11}$, constitue un accroc aux principes qui fondent l'éthique démocratique.

Nous étudierons ici la possibilité d'une critique conservatrice de l'éthique démocratique à travers l'oeuvre emblématique de Gérard Bouchard. Nous procèderons à une remise en question de l'obsession éthique qui commande la compréhension du fait politique et de l'histoire nationale. II s'agira d'illustrer la rupture entre une certaine approche normative du fait politique et des vérités fondamentales qui percent et invalident une exigence éthique trop serrée. Nous proposerons aussi une remise en question du langage politique contemporain, encore une fois dans son rapport trop intime à une éthique démocratique radicale, mais aussi en fonction de sa capacité à traduire le réel dans le langage. La question identitaire peut être éclairée par l'expérience historique de la Révolution tranquille. Nous tenterons ainsi de saisir comment la pensée politique québécoise contemporaine tente de décréter une nouvelle grande noirceur, celle des temps odieux de la «nation ethnique», dont le Québec serait en train de sortir péniblement pour permettre la réalisation intégrale d'une certaine éthique de la diversité supposée capable de réconcilier l'unité du récit mémoriel québécois et la diversité des trajectoires identitaires qui se déploient malgré l'homogénéité réductrice des récits collectifs acceptés.

\section{Gérard Bouchard ou la reconfiguration de l'identité nationale selon le paradigme de la post-modernité}

L'homme hanté par la mauvaise conscience ferait n'importe quoi pour racheter ses erreurs passées.

Chantal Millon-Delsol

10. James Tully, Une étrange multiplicité, le constitutionnalisme moderne à une époque de diversité, Québec, PUL, 1998, p. 5.

11. La République française est l'objet unanime des critiques des tenants de l'éthique de la pluriethnicité. 
La démarche sociologique, historique, et finalement politique ${ }^{12}$ de Gérard Bouchard repose sur un postulat clairement explicité : la "post-modernité» ouvre la porte à un questionnement radical quant aux identités collectives et aux narrations identitaires totalisantes qui prétendaient synthétiser l'expérience historique des peuples dans une conception unifiée et homogénéisante de la nation. "La crise de la conscience collective est au cœur de la postmodernité13». La nation n'est plus ce qu'elle était, d'ailleurs, elle n'a jamais été ce qu'elle prétendait être ${ }^{14}$.

Les collectivités d'hier étaient-elles donc si cohérentes? II faut se garder ici d'une illusion qui nous ferait voir les nations anciennes comme robustes et unifiées, homogènes et solidaires. Ce serait oublier qu'elles tenaient surtout par l'action autoritaire de l'État - même libéral - et des classes qui le dirigeaient 15 .

L'époque présente permet enfin un questionnement radical quant aux fondations explicites et implicites du fait national en tant que représentation généralement admise du vivre-ensemble. La postmodernité sonne le glas des méta-récits englobants. Autrement dit, il ne sera plus possible, nous dit Bouchard, de penser la nation comme une réalité historique vivante, dotée d'une volonté propre, d'une densité historique significative et existentielle. La nation est une construction identitaire strictement artificielle et elle doit se méfier de toute tentation essentialiste qui tenterait d'en faire de nouveau un mythe, au sens où pouvait l'entendre Georges Sorel, au sens où l'entendait aussi le général de Gaulle dans sa philosophie nationaliste

12. Comme le remarque avec justesse Jocelyn Létourneau dans Jocelyn Létourneau,

Passer à l'avenir, Montréal, Boréal, 2000, p. 43-78. Critiquant la téléologie indépendantiste qui animerait la démarche bouchardienne, Létourneau affirme ainsi que la réinterprétation de l'expérience historique québécoise "s'enracine davantage dans une préférence d'ordre politique qui surdétermine et infléchit toute la démarche" ( $p$. 46). Éric Bédard, représentant de la nouvelle sensibilité en histoire, voit plutôt dans le projet bouchardien une autre légitimation du grand récit de la Révolution tranquille, récit salvateur qui voit le Québec sortir de l'obscurantisme clérico-nationaliste pour entrer de plein pied dans une modernité longtemps étouffée au niveau des élites. Éric Bédard, "Genèse des nations et cultures du nouveau monde, le Magnum opus de l'historiographie moderniste" in Bulletin d'histoire politique, vol. 9, no 2, 2001, Comeau-Nadeau, p.160-174.

13. Gérard Bouchard, «Qu'est-ce qu'une nation?» op. cit., p. 477.

14. L'homogénéité apparente de la forme classique de l'identité nationale ne serait autre chose que le fruit d'une démarche autoritaire de la part de certaines élites qui auraient imposé pour des besoins spécifiques et souvent classistes, une représentation de la nation qui légitimait leur position sociale et politique.

15. Gérard Bouchard, "Qu'est-ce qu'une nation?» op. cit., p. 478. 
de l'histoire universelle et de l'histoire de France. Qu'on ne se fasse plus d'illusion, nous dit Bouchard, la nation elle aussi a été désenchantée, et les ingénieurs identitaires peuvent dorénavant s'engager dans une critique de l'imaginaire national selon des postulats éthiques ou épistémologiques, c'est selon. Ils peuvent même réconcilier ces deux démarches, comme le fait Bouchard, et proposer une nouvelle synthèse de l'histoire nationale comme mode spécifique de narration du vivre-ensemble à l'heure de la post-modernité identitaire. Ce qui est clair, c'est que la nation est de moins en moins un vivre-ensemble existentiellement et historiquement défini par l'adhésion implicite et spontanée de ses membres à la mémoire qu'elle propose en héritage, et de plus en plus un objet à géométrie variable offert aux sciences sociales qui peuvent le décomposer et le recomposer au gré des exigences éthiques, au nom de la démocratie, de la pluriethnicité, et pourquoi pas, de la démocratie dans un cadre pluri-ethnique. La nation cesse d'être considérée pour elle-même, pour devenir un jouet entre les mains des savants spécialistes de la question nationale, ceux que Serge Cantin appelle très justement les «universitaires spécialistes de la question nationale». Gérard Bouchard est explicite en cela :

Grâce aux recherches historiques et anthropologiques effectuées depuis vingt ou trente ans, nous savons bien que les identités et les cultures nationales ne sont pas des essences, qui se transmettraient intactes à travers et au-delà des générations et qui s'imposeraient aux contemporains en vertu d'une sorte de transcendance. Nous avons appris, au contraire, que ces objets culturels sont des construits de l'histoire; ils sont par conséquent soumis à ses aléas, à ses contingences ${ }^{16}$.

Ce constructivisme identitaire est sans appel. II n'y a aucune transcendance propre à l'expérience nationale. Les sciences sociales déconstruisent le mythe et sa prétention à dire l'histoire dans son propre langage. II est révélateur d'une critique que Robert Legros, à la suite d'Alain Finkielkraut, adresse à la formulation contemporaine de l'impératif démocratique :

Rien ne peut plus être reconnu comme un donné, qui ne doive être interprété immédiatement comme le produit d'une fabrication humaine. Rien ne peut plus apparaitre comme une dimension ou une composante de l'expérience humaine, qui ne doive

16. Gérard Bouchard, «Qu'est-ce qu’une nation?» Op. cit., p. 476. 
se comprendre comme le résultat d'une construction humaine.

Rien ne peut plus être considéré comme relevant de la nature qui ne doive être considéré comme un produit humain ${ }^{17}$.

Ce refus radical de toute essentialisation consciente de l'identité conduit cependant Bouchard à récuser toute forme d'héritage qui se présente sous la forme du donné. Le monde n'est pas un héritage à s'approprier, mais un processus à expliciter, un ensemble de représentations conscientes et inconscientes que doit recomposer à sa guise le spécialiste de l'identité nationale, détenteur de la nécessaire culture savante ${ }^{18}$ pour se pencher sur ces questions. On peut donc voir ici une technicisation de l'identité nationale, où sa juste interprétation dépend du discours légitime de quelques-uns, et pourquoi pas, d'un seul, qui perce la fausse conscience identitaire associée à l'interprétation traditionnelle de l'imaginaire national, dans son projet démiurgique de "bâtir le nouvel imaginaire historique des siens ${ }^{19}$ ". La nation devient le champ réservé des sociologues, historiens et spécialistes respectueux de l'éthique démocratique de la pluriethnicité. Ceux qui proposent un certain sens de la filiation, comme l'a fait Fernand Dumont, se replient sur une conception inacceptable du fait national, parce qu'il ne repose pas a priori sur une définition démocratique de celle-ci.

II n'y a aucun donné. La réalité est processuelle. II ne s'agit pas de reconquérir un héritage laissé en suspens par les générations précédentes, un héritage précédé d'aucun testament, comme aimait le rappeler Hannah Arendt, citant René Char, mais bien de réengager la compréhension d'hier selon aujourd'hui, de partir du présent pour choisir son passé, pour le reconstruire délibérément, pour le réécrire à l'aune de l'enseignement indépassable des sciences sociales et historiques. "Le passé m'indiffère si je ne l'ai pas choisi» (Vaneigheim). Bouchard livre un aperçu presque poétique de cette réinvention identitaire qui renonce à toute filiation existentiellemment perçue par la nation :

Le Québec trouverait sa vocation naturelle en récusant les chimères des deux mondes qui de toute manière lui échappent et en se constituant comme culture des interstices, en recherchant son monde interlope par des chemins obliques. Un tiers-

17. Robert Legros, "Penser notre humanité comme une énigme» in Esprit, Novembre 2001, 11, p.177.

18. A plusieurs reprises, dans son oeuvre, Bouchard déplore le traitement réservé à la culture savante au Québec.

19. Jocelyn Létourneau, op. cit., p.52 
monde en effet, mais moins par sa pauvreté que par son excentricité. En sorte que la perte et le deuil de ses deux univers de référence en fassent non pas un orphelin, qui serait voué éternellement au commentaire nostalgique et stérile d'une privation ou d'une absence, mais carrément un bâtard : ensauvagé comme au début (...), s'abreuvant à toutes les sources proches ou lointaines, mêlant et dissipant tous ses héritages, répudiant ses ancêtres réels, imaginaires et virtuels, il s'inventerait dans cette position un destin original qu'il pourrait enfin tutoyer, dans l'insouciance des ruptures et des continuités. Non pas un bâtard de la culture, mais une culture, et pourquoi pas, un paradigme du bâtard20.

Bouchard n'invite pas ceux dont il réinterprète le destin à renouer avec une filiation existentiellement définie par l'expérience historique du peuple d'ici, mais bien à inventer un destin, fondé sur la récusation de tous les héritages, sur la négation des ancêtres réels (dans notre cas, les premiers colons de la Nouvelle-France) pour embrasser les ancêtres imaginaires et librement choisis (dans ce cas, les Amérindiens). II s'agit d'une recomposition complète de l'imaginaire national selon l'éthique démocratique, où tout ce qui n'est pas choisi est récusé, où tout ce qui est hérité est déconstruit, où tout ce qui est représenté est éclaté.

Bouchard propose une dichotomie nouvelle pour surpasser la distinction usée entre la nation ethnique et la nation civique. II s'agirait ainsi de penser le phénomène des identités collectives à travers la dichotomie homogénéité/hétérogénéité. Cette dichotomie en cache une autre, qui se révèle ici et là, dans les replis du texte, mais qui n'est jamais clairement explicitée, qui n'est jamais formalisée. On la présentera ainsi : autoritarisme/démocratie. La conception homogène de la nation est nécessairement autoritaire, parce qu'elle voile une diversité sociologique indéniable que les sciences sociales ont depuis longtemps démontrée, telle une évidence. La conception homogène consacre les intérêts de quelques-uns qui, consciemment, proposent un discours relatif à l'identité nationale qui ne tient nullement compte des voies dissonantes qui se feraient nécessairement entendre si les interprètes officiels de la conscience nationale se mettaient à l'écoute de la diversité irréductible du social. Pour Bouchard, il n'y a jamais autre chose qu'un discours de l'homogénéité. Peut-être aussi des

20. Gérard Bouchard, Genèse des nations et cultures du nouveau monde, Montréal, Boréal, 2000, p.182 
pratiques uniformisantes, homogénéisantes, qui au nom de l'universalisme, qui au nom du nationalisme, tentent d'étouffer la diversité dans un projet plus ou moins avoué de construction identitaire. Mais toujours, la diversité refait surface et vient invalider la prétention autoritaire tendant à instituer une certaine forme de monolithisme identitaire. La République française doit affronter à la fois les régionalismes renaissants comme en témoigne le récent débat quant à la reconnaissance des langues régionales. Elle connaît aussi depuis maintenant vingt ans un problème immigrant, qui révèle l'échec partiel du modèle d'intégration universaliste et assimilationiste à intégrer au projet républicain les vagues maghrébines qui s'installent dans le sud de la France. La réapparition de la diversité apparaît aussi aux ÉtatsUnis, par le mouvement chicanos qui laisse voir dans un horizon politique plus ou moins rapproché l'hispanisation des États du sud longeant la frontière mexicaine. Autrement dit, Bouchard voit là la fin du melting pot américain.

Toutes ces démarches, aspirant à réaliser politiquement par le biais du pouvoir étatique l'homogénéisation de la nation, révèleraient une conception autoritaire de celle-ci. À cela, Gérard Bouchard offre un modèle alternatif, démocratique, parce que respectueux de la polyphonie irréductible du réel. On perçoit assez rapidement le fait que chez Bouchard, la démocratie est inséparable d'une reconnaissance explicite de la diversité culturelle. La démocratie n'est pas un simple régime politique, mais elle doit commander à la recomposition symbolique de la nation. On en revient donc à notre intuition initiale, lorsque nous avancions que la radicalisation de l'éthique démocratique est le fait politique majeur de notre temps. Lorsque la dynamique égalitaire et individualiste remet en question les formes élémentaires du donné, comme la nation et la culture, on ne peut faire autrement que de qualifier ce mouvement de radicalisation. Une perspective critique dirait même qu'il s'agit d'une tentation hégémonique propre à la dynamique du principe démocratique. Bouchard

21. Anthony Giddens, op. cit., p.70. Giddens définit ainsi le nationalisme xénophobe : "Xenophobic nationalism is the opposite (de la nation cosmopolite) : the nation is one, indivisible. It is culturally protectionist, assuming the nation have a destiny (...). But nations don't have destinies, and all nations, without exception, are "mongre/" nations. The nation is not something given in nature, and whatever remote connections they may have to earlier ethnic communities, nations are product of relatively recent history. They have all been built from a diversity of cultural fragments" (p.130-131). On peut imaginer facilement ce que Giddens entend alors lorsqu'il pose la démocratisation de la nation comme une exigence de sa troisième voie. 
applique ici une exigence que Anthony Giddens a lui-même formulée dans ses propres termes quant à la réinterprétation du phénomène national : la nation devient elle-même cosmopolite ${ }^{21}$. II reste une culture nationale chez Bouchard, on ne saurait en douter. Mais elle demeure "minimale»22, modeste, et laisse sa place à l'articulation d'une diversité fondamentale et indépassable. L'heure des identités démocratiques est venue. Contester cela est illusoire, nous dit Bouchard. Tocqueville nous l'aurait dit aussi. La progression du principe démocratique ne saurait être contenue, même pas par la tradition, même pas par la culture. La nation, qui a rendu la démocratie possible, comme le rappelle Alain Finkielkraut, est elle-même grugée par le principe démocratique, du moins dans la représentation officielle qu'en proposent les élites en place, soucieuses de proposer une imagerie nationaliste respectueuse des exigences éthiques qui articulent la pensée politique et la philosophie identitaire contemporaine. La nation était hier le siège possible de l'expérience démocratique. Comme le rappelle Philippe Bénéton, «la démocratie libérale requiert une substance extérieure à elle-même : une mémoire commune, des références partagées, la conscience d'un destin commun ${ }^{23}$ ". Robert Legros formule le même principe : «la nation est la condition de la démocratie 24 ». Si on pense la démocratie autrement qu'à travers sa forme procédurale, dans un souci républicain et patriotique explicite, on doit présupposer une conscience historique prédémocratique, un contexte où la démocratie peut germer. "Une argumentation ne pourrait jamais commencer et encore moins se poursuivre sans prémisses particulières, sans accords tacites, sans références implicites, sans une manière particulière d'entendre les lieux communs, bref sans une culture25». Alain Finkielkraut reprend aussi cette idée d'un lien indépassable entre la communauté de sens et la démocratie politique. "C'est dans le cadre national que l'unité du peuple peut s'accorder le plus facilement avec la pluralité des opinions 26 ”. Or ni chez Mill, ni chez Legros, ni chez Finkielkraut, la nation elle-même n'est soumise à une entreprise de refondation démocratique. On ne peut tout soumettre à la discussion, à la réinterprétation, il faut une base d'indiscuté, une mise en commun initiale qui rend possible la

22. Gérard Bouchard, "Qu'est-ce qu'une nation?», op. cit., p. 492.

23. Philippe Bénéton, Introduction à la politique, PUF, 1997, p. 356.

24. Robert Legros, op., cit. p.176.

25. Robert Legros, "Nation et démocratie" in Le messager européen, no 6, 1992, p. 23.

26. Alain Finkielkraut, L'ingratitude, Québec-Amérique, 1999, p. 57. 
démocratie. Explorant la tension entre la nation et la démocratie, Robert Legros voit dans l'une la condition de possibilité de l'autre, et dans l'autre la condition d'une dénaturalisation de l'une :

II n'est pas douteux que nation et démocratie recèlent des exigences divergentes. Qu'une nation démocratique soit par principe tiraillée par des tendances diverses, et traversée par des aspirations qui ne convergent pas. Mais des exigences divergentes ne pourraient-elles s'accomplir qu'en se dissociant. Leur association ne pourrait-elle être la condition de leur survie?27

II apparaît illusoire de soumettre l'identité nationale à une délibération exhaustive, ou celle-ci serait spécifiquement créée. Comme le rappelle Joël Roman, même lorsque Renan définit la nation dans le cadre de la modernité, il ne peut faire l'économie "d'une tradition héritée et non réfléchie, qui relève d'habitus constitués et non d'une délibération ${ }^{28 \% \text {. }}$

La conception dumontienne de la nation «française" du Québec est animée par la même idée d'un sentiment d'appartenance intuitif qui ne saurait être dévoilé entièrement par la raison, celle-ci serait-elle soumise à l'impérieuse éthique démocratique de la pluriethnicité :

Nous ne percevons pas une culture au sens où nous percevons un objet. II faudrait dire plutôt que nous en avons l'expérience. Cette expérience est pourtant magique, et plus encore peut-être que la perception. Nous parlons spontanément du Québec comme d'une globalité qui a un sens. Pourtant, nous n'en savons pas le détail, ni même les éventuels éléments significatifs. Nous ne disposons pas de tous les indices démographiques, économiques, historiques, etc.; nous n'avons pas éprouvé personnellement ce que cela signifie d'exister dans tel ou tel quartier de Montréal, dans la Beauce, dans Charlevoix, sur la Côte Nord ou en Abitibi. Le sentiment d'appartenance est global parce qu'il est magique29.

Cette magie de l'appartenance, qui s'exprime dans un langage modeste devant l'énigme du réel, Dumont ne tente pas de l'expliciter intégralement, non plus que de la nier. II l'accepte pour ce qu'elle est, une réalité dont le langage nous permet de saisir l'existence sans en dévoiler la substance intime. Le monde ne se réduit pas à ce qu'en

27. Robert Legros, «Nation et démocratie», op. cit., p.13.

28. Cité par Robert Legros, «Nation et démocratie», op. cit., p.14.

29. Fernand Dumont, Le sort de la culture, L'Hexagone, 1987, p. 318. 
disent les sciences sociales, non plus que l'histoire, même si on la rebaptise «science historique»30. Dans sa Genèse de la société québécoise, Fernand Dumont fournit un concept qui permet de penser cette magie sans la soumettre à un rationalisme excessif. On parle bien sûr de la référence dumontienne ${ }^{31}$, sensée donner un aperçu de l'intuition identitaire avant sa mise en discours systématique. À la recherche d'une anthropologie de l'évidence identitaire, Dumont souhaite "une autre science (qui s'intéresserait) au moment d'avant la représentation ${ }^{32}$ ». Le contact avec le langage dumontien, langage de la modestie face à l'héritage, autrement dit, langage de l'héritier plutôt que du fondateur, d'un langage intime avec la sinuosité du réel, illustre une des failles majeures du projet bouchardien. Prisonnier d'une certaine arrogance propre aux sciences sociales, Bouchard pense pouvoir dire la totalité du monde dans le langage des sciences sociales. II n'y a rien de sacré, il n'y a aucun tabou qui résiste à la refondation démocratique de la nation à l'heure de la diversité. II n'y a aucune magie de l'appartenance. II s'agit de déconstruire le réel pour le recomposer par la suite selon les impératifs éthiques et les postulats épistémologiques propres à la réécriture intégrale de l'imaginaire national. D'ailleurs, ce pari déconstructionniste, Bouchard le revendique comme tel :
L'une des caractéristiques de la culture nationale, c'est de ne pas être innocente; c'est pourquoi les entreprises de déconstruction sont nécessaires, du moins, à titre préalable, pour éclairer les démarches de reconstruction. Ces remarques veulent attirer l'attention sur ce qu'on pourrait appeler l'ingénierie identitaire et sur la nécessité d'en démocratiser l'usage ${ }^{33}$.

Cette déconstruction, cette démystification de la magie de l'appartenance, Bouchard en voit la possibilité dans les sciences sociales, plus spécialement dans l'histoire sociale du phénomène national. Sous prétexte de connaître le tout du vivre-ensemble, il s'agit de dévoiler l'intimité de l'appartenance et des modes d'appartenance par une rationalité froide susceptible de débusquer la fausse conscience qui masquerait l'hétérogénéité radicale de la culture nationale :

Grâce à l'histoire sociale, il est plus facile de mettre à jour les feintes et les subterfuges de l'imaginaire national, tout comme

30. Comme le faisait aussi Althusser, on remarquera.

31. Fernand Dumont, Genèse de la société québécoise, Boréal, 1993, p.337-352.

32. Fernand Dumont, Genèse de la société québécoise, Boréal, 1993, p.339.

33. Gérard Bouchard, "Qu'est-ce qu'une nation?», op. cit., p. 476. 
ses contradictions, omissions et distorsions. Deviennent également plus manifestes les diverses formes de discrimination et d'exclusion, de divisions et de clivages, qui relèvent aussi de l'histoire nationale ${ }^{34}$.

Le réel est décodé. Le mythe ne survit pas à l'investigation scientifique qui révèle objectivement la nature de l'identité nationale. Le seul langage qui convient pour dire le vrai sur l'identité est celui de la science sociale, qui est propre d'ailleurs à une traduction ultérieure selon les catégories de l'éthique afin de jouer un rôle pédagogique et civique dans l'éducation à la citoyenneté de la population québécoise. L'idée de nation est inféodée, cela va de soi, aux principes universels révélés par l'éthique comme science de la justice. Le langage politique au terme de la démarche bouchardienne doit être conforme "à la science historique lorsqu'elle veut remplir une fonction civique et s'adresser à l'ensemble d'une société pluraliste 35 ». Un critique dirait de la démarche bouchardienne qu'elle est à proprement parler démiurgique. Refonder la nation, intégralement, après l'avoir déconstruite par le biais du discours révélateur des sciences sociales, démystifier la magie de l'appartenance, la soumettre à cette science de la justice qu'est l'éthique démocratique de la pluriethnicité, on peut aisément dire de Bouchard qu'il formule un "vaste programme» qui se veut explicitement fondateur d'un nouvel imaginaire national délesté des représentations anciennes de la conscience historique québécoise. Cette démocratisation de la nation, légitimée par la démarche scientifique propre aux sciences sociales, se veut non seulement juste, mais prétend aussi être vraie.

II s'agit de voir maintenant si la totalité du monde peut être dite dans le langage de la science sociale. L'ingénierie identitaire qu'on retrouve au fil de l'oeuvre bouchardienne est-elle à même de penser le phénomène national dans un langage qui lui convient?

\section{L'obsession éthique ou le rapport biaisé à la réalité}

Le saisie conceptuelle du monde est précédée dans la démarche bouchardienne d'un présupposé éthique qu'on a jusqu'ici qualifié de démocratique. L'impérieuse démocratisation de l'identité nationale exige donc une lecture du réel qui corresponde au résultat attendu, soit une nation conforme au "modèle de la nation québécoise" que Bouchard formule et précise au fil de ses nombreuses publications.

34. Gérard Bouchard, La nation québécoise au futur et au passé, VLB, 1999, p.100.

35. Gérard Bouchard, op. cit., p.139. 
Cette dérive de la pensée contemporaine, Gérard Bouchard n'en a pas le monopole. Il semble que l'obsession éthique, le souci du juste, le souci, disons-le, d'une science de la justice, précède toute compréhension de la réalité pour elle-même, toute compréhension de l'événement, de l'histoire, toute appropriation de la conscience historique de la nation à laquelle on participe. Chez Dumont, la mémoire était à l'origine de la méthode ${ }^{36}$. Chez Bouchard, c'est l'éthique de la pluriethnicité entendue au sens d'une éthique de la démocratie en contexte d'hétérogénéité, qui est à l'origine de la méthode. Chez Bouchard, en plus, ce primat de l'éthique sur le réel (qui conduit par exemple l'historien à déconstruire le réel pour le rebâtir ensuite selon les postulats éthiques qu'il adopte) se double d'une prétention des sciences sociales à la saisie de la totalité du réel. Bouchard devient ainsi un scientifique de l'identité, le détenteur d'une science de la nation, qu'il baptise histoire nationale, mais qui est en vérité la réécriture de l'histoire de la nation selon une éthique étrangère à la conscience historique intuitive qui anime la communauté nationale.

II n'est pas dit cependant que cette appréhension du monde soit à même de nous offrir un rapport fructueux avec le réel. II n'y a pas d'identité radicale entre le discours et la réalité, entre l'intuition identitaire du pays et la formalisation de cette identité dans le langage des sciences sociales. Le réel est sinueux. II est parsemé d'énigmes que la conscience ne peut esquiver sous prétexte qu'elle ne peut en dévoiler l'équation conceptuelle fondamentale. Lorsque Dumont dit de l'identité qu'elle est magique, il démontre sa nette conscience des insuffisances du langage pour dire la totalité du monde et fait appel à un mot évocateur qui, sans prétendre à l'identité du réel et du concept, donne un aperçu sur une réalité indéniable qui ne relève toutefois pas nécessairement de la science. Cette modestie du langage, cette conscience de l'indicible, cette modestie des aspirations, n'appartiennent pas au projet bouchardien. Son rapport au réel en est par conséquent biaisé, fragilisé qu'il est en plus par le jugement normatif inscrit au sein même de sa compréhension de la nation dans son identité historique. En refusant d'assumer ne serait-ce qu'une partie de l'interprétation traditionnelle de l'histoire nationale canadiennefrançaise/québécoise, Bouchard propose une compréhension qui est en évidente rupture avec la filiation historique constitutive de l'identité nationale. Or, dans l'éventualité où ce discours ne serait plus l'apanage de quelques savants de cabinet, mais s'installerait au sein de

36. Fernand Dumont, Le sort de la culture, op. cit., p.318. 
l'imaginaire national, c'est à une nouvelle opération de la table rase, cette fois identitaire, que serait invitée la nation québécoise. Chantal Millon-Delsol formulait une critique vive de cette attitude qui consiste à se replier dans un univers linguistique clairement idéologique pour ne pas affronter la réalité. "Un peuple qui défigure le réel parce qu'il n'a pas envie de se trouver en face de la vérité nue devient vite la victime effarée de ses propres élucubrations ${ }^{37}$ ". Cette fuite en avant, qui consiste à refuser le réel au nom d'une éthique de la diversité sacralisée, amène l'historien à réécrire complètement l'histoire nationale, en rupture bien souvent avec la façon dont celle-ci est interprétée dans l'imaginaire national38.

Il y a un peu de cela dans la méthode de Gérard Bouchard. Parce qu'il considère l'identité nationale comme quelque chose de purement artificiel, de strictement conventionnel, parce qu'elle n'est pour lui qu'un discours susceptible d'être aménagé telle une nation à géométrie variable, il peut librement proposer un nouveau langage en pensant ainsi proposer une nouvelle nation. Puisque la juste interprétation de la nation est le monopole des scientifiques de l'identité nationale, il s'agit de proposer une interprétation contre une autre, un modèle contre un autre, pour changer d'un coup l'identité nationale. La nation des universitaires apparaît ainsi comme un jouet conceptuel, que peuvent manier à loisir les nouveaux ingénieurs identitaires, identiques tant dans leurs méthodes que dans leurs errements avec les ingénieurs sociaux d'hier. Bouchard est prisonnier de l'éthique démocratique, qui commande une lecture du fait national selon le paradigme de l'hétérogénéité, et d'une épistémologie constructiviste qui présuppose le caractère construit de tout ce qui est humain. L'une ne va pas sans l'autre. Bouchard refuse de considérer qu'on puisse savoir quelque chose de vrai sur le monde en dehors de sa méthode scientifique. La conscience identitaire et historique du peuple québécois doit être tamisée par cette science sociale qu'est la science historique :

Récusant toute forme de positivisme, je crois que science et conscience vont de pair. Mais je me méfie de la conscience qui

37. Chantal-Millon Delsol, La politique dénaturée, PUF, 1988, p.17.

38. Gérard Bouchard critique d'ailleurs la majorité francophone, décelant chez elle des relents d'autoritarisme, dans son refus de s'effacer devant la mosaïque ethnique à laquelle s'apparente la nation co-intégrée du professeur Bouchard. «À certains signes, en effet, il semble parfois que la population francophone de souche n'a pas complété son apprentissage dans le rôle d'une majorité démocratique, sensible à la réalité interculturelle». Gérard Bouchard, La nation québécoise au futur et au passé, op. cit., p.77. Nous rappelons ici que ce n'est pas Pierre Elliot Trudeau qui parle, mais Gérard Bouchard. Au cas où les derniers propos nous auraient égarés... 
ne se soumet pas intégralement à l'épreuve de la science ${ }^{39}$.

En son temps, Friedrich von Hayek a critiqué vivement les ingénieurs sociaux qui pensaient la réalité sociale comme une mécanique analogue à un plan rationnel, à un plan d'ingénieur, qu'il suffisait de reprogrammer en modifiant l'équation au centre de la dynamique sociale pour la rendre plus conforme à une certaine éthique de la justice, autrefois définie dans sa proximité avec l'idéal socialiste. Le réel serait rationnel, et le rationnel serait réel. II n'y aurait ainsi aucune limite à la refondation infinie de la réalité si on lui plaquait très justement un plan d'ensemble révélateur de son détail et des procédures commandées pour effectuer le changement entrevu à l'horizon. Dénonciateur du constructivisme en science sociale, Hayek critiquait ceux qui pensaient pouvoir recréer la société à partir d'un projet de société théorique.

À l'ingénierie sociale a succédé, on le voit, l'ingénierie identitaire, qui postule une complète malléabilité des identités collectives, où les spécialistes de la nation peuvent librement reconfigurer l'imaginaire national en faisant l'économie de l'interprétation qu'en propose la tradition. Encore une fois ici, on postule la possible reconstruction du monde à partir d'une vision du monde. On postule la réinvention de la nation au nom d'un nouveau «modèle de la nation québécoise». Et au diable la nation réelle! Nation réelle qui, en plus, n'est qu'une chimère, car une nation n'est jamais qu'un aménagement temporaire de différents rapports de forces identitaires au sein d'une société diversifiée. C'est un peu là la leçon de Bouchard.

\section{Une nouvelle Révolution tranquille identitaire}

L'apparition récente du discours sur la nation civique, ou du moins, de l'ouverture théorique à une certaine vision éclatée de la nation québécoise dans la compréhension officielle de son identité historique prend de plus en plus le visage de la dichotomie grande noirceur/Révolution tranquille qui a structuré l'imaginaire historique québécois depuis l'aube des années soixante. 1960 était notre première année zéro. Une deuxième année zéro, dont on fixera un jour l'apparition précise entre 1995 et l'année 2001 sert désormais de point de référence au discours sur la nation québécoise. Le passage de l'homogénéité à l'hétérogénéité, pour reprendre les catégories de

39. Gérard Bouchard, «Réplique au texte de Serge Cantin, Sur le modèle de la nation québécoise et la conception de la nation chez Fernand Dumont" in Bulletin d'Histoire politique, vol. 9, no 2, printemps, 2001, p.154-155. 
Bouchard, est analogue à la sortie d'un obscurantisme identitaire qui niait la diversité culturelle de la société québécoise pour y plaquer une homogénéité de papier. Avant, la nation "ethnique», maintenant, la nation "civique». Avant, l'exclusion, maintenant, l'inclusion. Avant, Lionel Groulx, maintenant, Gérard Bouchard. Et autant qu'hier on mangeait du curé, maintenant, on mange de l'ethnique. Avant, on traquait les vestiges du clérico-nationalisme. Maintenant, on chasse les derniers relais d'ethnicisme. La même interprétation révolutionnaire des mutations historiques qui affectent l'imaginaire québécois est proposée. Incidemment, avec la même volonté de faire table rase d'un passé qu'on identifie à l'obscurantisme. La norme qui permet de décréter cette césure est l'éthique démocratique comprise comme une éthique de la pluriethnicité. Nous ne sommes plus les fils d'un hier canadien-français, mais bien d'une nation spontanée, qui sera, dans la perspective indépendantiste, la nation fondatrice du Québec. Plus d'hier ni de demain, qu'un aujourd'hui dont il s'agit d'accroître la profondeur en révélant toujours davantage sa fragmentation infinie. L'affaire Michaud a bien illustré ce possible dérapage d'un nationalisme civique qui, au nom de l'éthique démocratique de la diversité, a entraîné la condamnation d'un militant nationaliste qui tenait à rappeler la filiation canadienne-française dans la définition du sentiment identitaire québécois. Elle a bien illustré aussi l'intolérance possible de l'éthique démocratique, non seulement face aux critiques antidémocratiques de celle-ci, mais simplement envers les considérations, qui ne se veulent pas a priori démocratiques, relatives à la définition de l'identité nationale.

Contre cette interprétation tronquée de l'identité nationale qui, en plus, intègre une césure révolutionnaire dans l'imaginaire collectif virtuellement porteuse d'intolérance, on peut opposer une critique conservatrice. Nous proposons ici une définition assez brève de ce conservatisme nécessaire : la conscience de la fragilité du donné, la volonté de préserver un héritage, de conserver une filiation entre les vivants d'hier et ceux d'aujourd'hui. Incidemment, une méfiance déclarée envers toute éthique qui ne puise pas d'abord dans la conscience d'une réalité particulière, réalité qui, la plupart du temps, prend le visage de la nationalité. Le vieil adage burkien garde toute sa pertinence : on ne peut ni ne doit se départir de ses préjugés dans la compréhension du destin de sa communauté particulière. Ses préjugés, autrement dit, ses traditions, sa mémoire, un ensemble de considérations qui ne puisent pas d'abord à une éthique rationaliste et démocratique et qui ne doivent pas pour autant être disqualifiées. Une 
méfiance nécessaire, aussi, envers ce constructivisme radical qui plaque sur toute l'expérience humaine un postulat d'artificialité. Un refus de plaquer sur le particulier des principes universels qui, sous le couvert d'une science du juste, d'une éthique en tant que science, nie la pluralité constitutive de l'humanité concrète et le caractère indépassable de ses nombreux visages. Une modestie du langage, qui ne croit pas pouvoir résumer la réalité dans un amalgame conceptuel aux prétentions scientifiques. Une ouverture du langage à la métaphore poétique comme interprétation légitime du réel. Le refus aussi d'enfermer le langage légitime dans le discours des sciences sociales.

Le progressisme et le démocratisme identitaire qui se déploient dans la société québécoise contemporaine, et dont Gérard Bouchard est la figure emblématique, nécessitent une critique qui ne puise pas au principe démocratique. Non pas une critique antidémocratique, mais bien une critique qui récuse l'universalisme radical de l'éthique démocratique. Une critique qui réaffirme la validité des consciences historiques particulières, contre ceux qui n'y voient que facticité. Une critique qui parle au nom du pays réel sans se laisser aveugler par le pays légal. Somme toute, une critique qui, au nom de l'histoire, révèle l'insuffisance d'un présent autarcique, d'un présent statique, qui se croit tout permis, qui se croit même permis de se choisir un passé pardelà l'évidence du passé réel. Gérard Bouchard dit du passé qu'il est imprévisible ${ }^{40}$. On peut lui répondre qu'il n'est imprévisible que dans la mesure où le présent le plus immédiat monopolise toutes nos préoccupations, dans la mesure où nous sommes saisis d'un violent "ethnocentrisme de l'actuel» (Alain Finkielkraut). II s'agit de proposer une nouvelle conscience de la filiation, où la démiurgie identitaire sera critiquée au nom du réel en lui-même, de sa densité historique, et d'une nette volonté de le préserver d'un langage qui n'existe plus dans le monde, qui se perd dans l'idéologie et qui ne traduit plus la conscience qu'ont les hommes de vivre une vie bien légitime, par-delà le jugement décrété des spécialistes des sciences sociales et des savants techniciens de l'identité éclatée.

\section{Mathieu Bock-Côté, Étudiant au baccalauréat philosophie Université de Montréal}

40. Gérard Bouchard, La nation québécoise au futur et au passé, op. cit., p.119. 